\title{
INVESTIGATION OF FEASIBILITY OF WIND TURBULENCE MEASUREMENT BY A PULSED COHERENT DOPPLER LIDAR IN THE ATMOSPHERIC BOUNDARY LAYER
}

\author{
Igor Smalikho $^{1 *}$, Viktor Banakh ${ }^{2}$ \\ ${ }^{1,2}$ Institute of Atmospheric Optics of Siberian Branch of the Russian Academy of Sciences, Russia, \\ *smalikho@iao.ru
}

\begin{abstract}
Feasibilities of determination of the wind turbulence parameters from data measured by the Stream Line coherent Doppler lidar under different atmospheric conditions have been studied experimentally. It has been found that the spatial structure of the turbulence is described well by the von Karman model in the layer of intensive mixing. From the lidar measurements at night under stable conditions the estimation of the outer scale of turbulence with the use of the von Karman model is not possible.
\end{abstract}

\section{INTRODUCTION}

Pulsed coherent Doppler lidars (PCDL) are used for the study of dynamic processes in the atmosphere, aircraft wake vortices and wind turbine wakes [1]. PCDLs can be also used for measurements of wind turbulence in the atmospheric boundary layer. A method of the azimuth structure function (ASF) for estimation of the turbulence parameters (radial velocity variance $\sigma_{r}^{2}$, turbulent energy dissipation rate $\varepsilon$ and integral scale of the turbulence $L_{V}$ ) from data measured with a conically scanning PCDL has been developed in $[2,3]$. In this method it is assumed that the spatial structure of the wind turbulence is described by the von Karman model. In this work we investigate of feasibility of application of the ASF method for wind turbulence measurement under different conditions of the atmospheric boundary layer.

\section{MEASUREMENT STRATEGY MODIFIED ASF METHOD}

AND

Conical scanning is performed at a fixed elevation angle $\varphi$, using rotation of the probing beam about a vertical axis $z$ with a constant angular speed $\omega_{c}$. During one scan the azimuth angle $\theta$ varies from 0 to $2 \pi$. The components of the instantaneous wind velocity vector $\mathbf{V}=\left\{V_{z}, V_{x}, V_{y}\right\}$ are random functions of coordinates $\mathbf{r}=\{z, x, y\}$ and time $t$. Due to the turbulence anisotropy, their variances $\sigma_{z}^{2}, \sigma_{x}^{2}$ and $\sigma_{y}^{2}$ differ from each other, and the radial velocity variance $\sigma_{r}^{2}$ depends on the azimuth angle $\theta$, where $\quad \sigma_{\alpha}^{2}=<\left(V_{\alpha}^{\prime}\right)^{2}>; \quad V_{\alpha}^{\prime}=V_{\alpha}-<V_{\alpha}>$; $\alpha=z, x, y, r ; \quad V_{r}=\mathbf{S} \cdot \mathbf{V} ; \quad$ and $\mathbf{S}=\{\sin \varphi, \cos \varphi \cos \theta, \cos \varphi \sin \theta\} . \quad$ For the elevation angle $\varphi=\tan ^{-1}(1 / \sqrt{2}) \approx 35.26^{\circ}$ the radial velocity variance averaged over the azimuth angles $\bar{\sigma}_{r}^{2}$ is related with the kinetic energy of turbulence $E=\left(\sigma_{z}^{2}+\sigma_{x}^{2}+\sigma_{y}^{2}\right) / 2$ as [4]

$$
E=(3 / 2) \bar{\sigma}_{r}^{2} \text {. }
$$

In this equation it is possible to take into account the averaging of the radial velocity over the sensing volume based on the theory developed in the monograph [1].

From raw lidar data we obtain an array of estimates of the radial velocity $V_{L}\left(\theta_{m}, R_{k}, n\right)$, where $\theta_{m}=m \Delta \theta$ is the azimuth angle; $m=0,1,2, \ldots, M-1 ; \Delta \theta$ is the azimuth angle resolution; $R_{k}=R_{0}+k \Delta R$ is the distance from the lidar to the sensing volume center; $k=0,1,2, \ldots, K ; \Delta R$ is the range gate length; $n=1,2,3, \ldots, N$ is the number of conical scan. Then for each height $h_{k}=R_{k} \sin \varphi$ we calculate the azimuth structure function $D_{L}\left(\psi_{l}\right)$ by the equation

$$
D_{L}\left(\psi_{l}\right)=\frac{1}{N} \sum_{n=1}^{N} \frac{1}{M-l} \sum_{m=0}^{M-1-l} B^{2}\left(\psi_{l}, \theta_{m}, n\right),
$$


where $\quad \psi_{l}=l \Delta \theta ; \quad l=1,2, \ldots, L$; $B\left(\psi_{l}, \theta_{m}, n\right)=V_{L}^{\prime}\left(\theta_{m}+\psi_{l}, R_{k}, n\right)-V_{L}^{\prime}\left(\theta_{m}, R_{k}, n\right) ;$ $V_{L}^{\prime}\left(\theta_{m}, R_{k}, n\right)=V_{L}\left(\theta_{m}, R_{k}, n\right)-\mathbf{S}\left(\theta_{m}\right) \cdot \overline{\mathbf{V}}\left(h_{k}\right) ; \quad$ and $\overline{\mathbf{V}}\left(h_{k}\right)$ is the lidar estimate of the mean wind velocity vector obtained with the use of the sinewave fitting [1]. According to the theory [1], the averaged lidar estimate of the structure function can be represented as $\left\langle D\left(\psi_{l}\right)\right\rangle=D_{a}\left(\psi_{l}\right)+2 \sigma_{e}^{2}$, where $D_{a}\left(\psi_{l}\right)$ is the azimuth structure function of the radial velocity averaged over the sensing volume and $\sigma_{e}$ is the instrumental error of the radial velocity estimate. If $R_{k} \cos \varphi>L_{V}$, the function $D_{a}\left(\psi_{l}\right)$ can be replaced by the transverse structure function $\quad D_{\perp}\left(l \Delta y_{k}\right), \quad$ where $\Delta y_{k}=\Delta \theta R_{k} \cos \varphi$.

Using the von Karman model for the 2-D spatial spectrum of wind velocity fluctuations and assuming that the turbulence is isotropic one can calculate the function $D_{\perp}\left(l \Delta y_{k}\right)$, which depends on two turbulent parameters: $\varepsilon$ and $L_{V}$ [3]. Taking into account that the Kolmogorov constant $C_{K} \approx 2$, for the von Karman model the integral scale $L_{V}$, the kinetic energy $E$ and the dissipation rate $\varepsilon$ have the following relation:

$$
L_{V}=0.38 E^{3 / 2} / \varepsilon \text {. }
$$

In [3] it is offered to estimate $\varepsilon$ and $L_{V}$ by the use of the least square method for the fitting of $\Delta D_{\perp}\left(l \Delta y_{k}\right)=D_{\perp}\left(l \Delta y_{k}\right)-D_{\perp}\left(\Delta y_{k}\right)$ to $\Delta D_{L}\left(\psi_{l}\right)=D_{L}\left(\psi_{l}\right)-D_{\perp}(\Delta \theta)$. However, if the condition $R_{k} \cos \varphi>L_{V}$ is not true, the error of estimate of the integral scale $L_{V}$ can be very large. To avoid this problem, we offer the following approach for estimation of the turbulence parameters (modified ASF method). First, from measured $\Delta D_{L}\left(\psi_{l}\right)$ we estimate the turbulent energy dissipation rate $\varepsilon$ within the inertial interval of the turbulence (when $l \Delta y_{k}<L_{V}$ ) [5]. Then, from the array $V_{L}^{\prime}\left(\theta_{m}, R_{k}, n\right)$ the radial velocity variance $\bar{\sigma}_{r}^{2}$ is calculated. Taking into account that the elevation angle $\varphi=35.26^{\circ}$, we estimate $E$ and $L_{V}$ by the Eqs. (1) and (3), respectively.

\section{EXPERIMENT}

To investigate feasibility of determination of the wind turbulence parameters from data measured by PCDL with the use of the conical scanning at the elevation angle $\varphi=35.26^{\circ}$, we carried out an experiment on the Basic Experimental Complex of the Institute of atmospheric Optics SB RAS from 21 to 23 July 2016. The continuous measurement by the Stream Line lidar (made by HALO Photonics) was conducted at $\omega_{c}=6^{\circ} \mathrm{s}^{-1}$ (duration of one scan $T_{\text {scan }}=1 \mathrm{~min}$ ) and $\Delta \theta=3^{\circ}$ ( $M=120$ ). During the entire experiment was clear weather. At night time always the stable thermal stratification took place. Vertical profiles of the mean wind velocity and turbulence parameters were retrieved from the measured lidar data with the height resolution of $10 \mathrm{~m}$. Each profile was obtained when for the averaging we used the raw lidar data measured for 30 scans $\left(N=30\right.$, duration of the measurement $N T_{\text {scan }}=$ $30 \mathrm{~min})$.

Figure 1 shows results of the spatiotemporal visualization of the mean wind velocity and the turbulence parameters: $E, \varepsilon$ and $L_{V}$. In this figure it is well visible contrasts of the turbulence intensity at night and daytime. At night, due to the stability of the thermal stratification, when a lowlevel jet stream was formed (in a layer at altitudes of $150-300 \mathrm{~m}$ ), the turbulence was very weak, and in the afternoon there was an intensive turbulent mixing. The data obtained for the kinetic energy and its dissipation rate are not contrary to the atmospheric boundary layer theory. Obtained using Eq.(3) the values of the integral scale of turbulence for the daytime (when the temperature stratification is neutral or unstable) are consistent with known experimental data [6]. However, in areas colored white in Figure 2(d) (areas where temperature stratification was stable), the integral scale of turbulence $L_{V}$ takes very large values, which contradicts clearly to the theory of turbulence of the atmospheric boundary layer.

To identify the reasons of the overestimation of the integral scale $L_{V}$ for the data measured by the lidar during nighttime, we compared experimental azimuth structure function $\bar{D}_{L}\left(\psi_{l}\right)=$ 


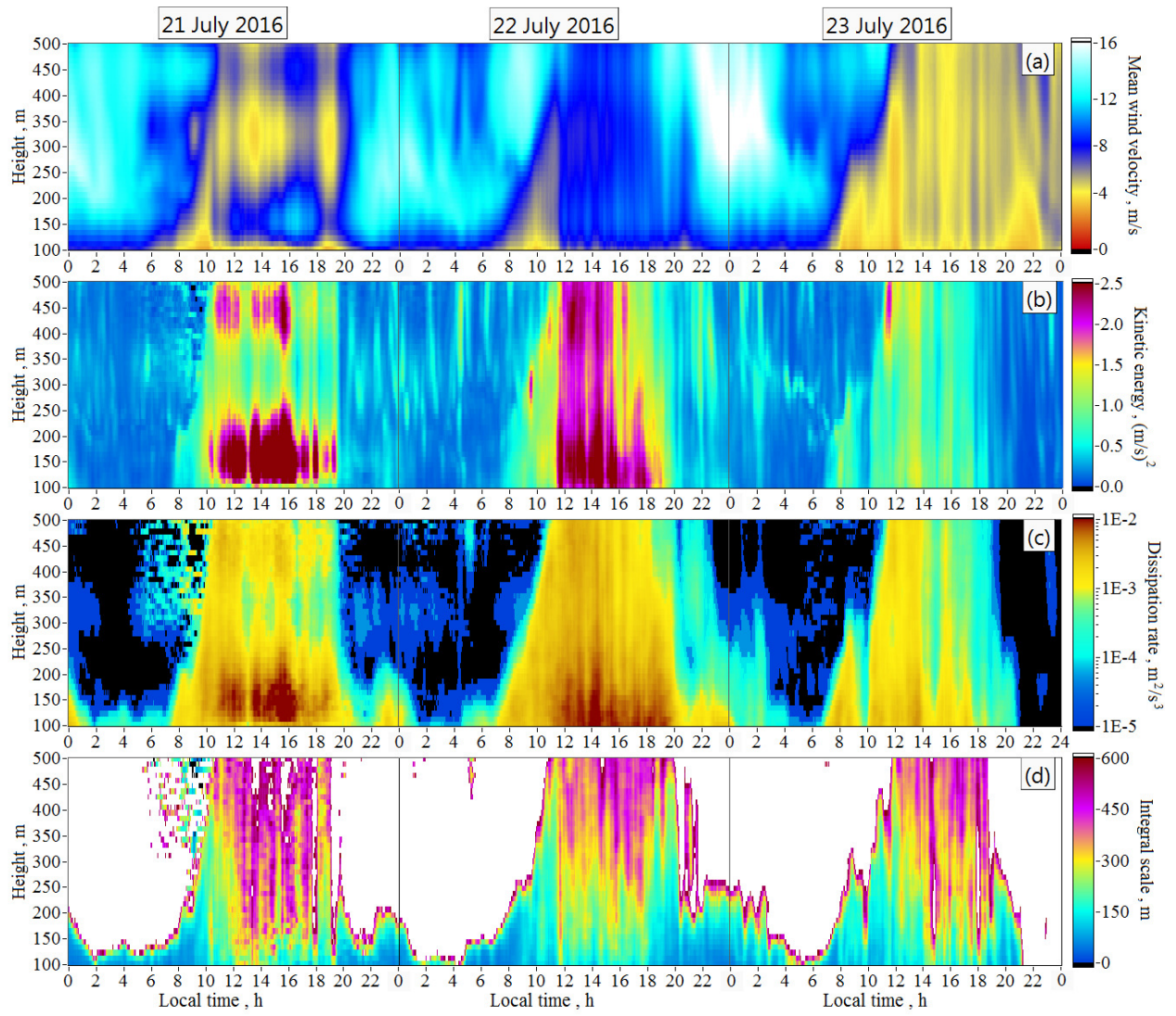

Figure 1 Spatiotemporal distribution of the mean wind velocity $(a)$, the turbulent kinetic energy $(b)$, the turbulent energy dissipation rate $(c)$ and the integral scale of turbulence $(d)$ obtained from measurements by the Stream Line lidar on the Basic Experimental Complex IAO SB RAS.

$D_{L}\left(\psi_{l}\right)-2 \sigma_{e}^{2}$ with the function $D_{\perp}\left(l \Delta y_{k}\right)$ which is calculated using the von Karman model and the obtained estimates of dissipation rate $\varepsilon$ and integral scale $L_{V}$. Figure 2 shows two examples of comparison of these functions for the cases of measurement at day and night. In Figure 2(a), there is an almost complete coincidence of the structure functions. Consequently, the turbulence structure is well described by the von Karman model and correct estimate of the integral scale of turbulence can be obtained using Eq. (3). The data in Figure 2(b) were obtained from lidar measurements at stable temperature stratification. In this case, the function $\bar{D}_{L}\left(\psi_{l}\right)$ greatly exceeds the $D_{\perp}\left(l \Delta y_{k}\right)$ at large spatial scales and we can conclude that for such conditions the turbulence structure is not described by the von Karman model. Accordingly, the correct estimate of the integral scale $L_{V}$ can not be obtained by Eq. (3).
To determine the areas for which the von Karman model is applicable, we calculated parameter characterizing the degree of divergence of structure functions $\bar{D}_{L}\left(\psi_{l}\right)$ and $D_{\perp}\left(l \Delta y_{k}\right)$ for different heights and time of day by the equation

$$
\gamma=\left\{L^{-1} \sum_{l=1}^{L}\left[\bar{D}_{L}\left(\psi_{l}\right) / D_{\perp}\left(l \Delta y_{k}\right)-1\right]^{2}\right\}^{1 / 2} .
$$

Parameter $\gamma=0.02$ for the data in Figure 2(a) and $\gamma=1.57$ for the data in Figure 2(b).

Figure 3 shows the spatiotemporal distribution of the parameter $\gamma$ for the data measured for a full day 07.22.2016. According to this figure, within a layer of $100-500 \mathrm{~m}$ and interval from 11:00 to 20:00 LT the parameter $\gamma$ varies from 0.01 to 0.2 (an average of about 0.1 ). This means that in this period of time, wind turbulence quite well described by the von Karman model and from the data measured by the conical scanning lidar one can obtain reliable information about the integral 

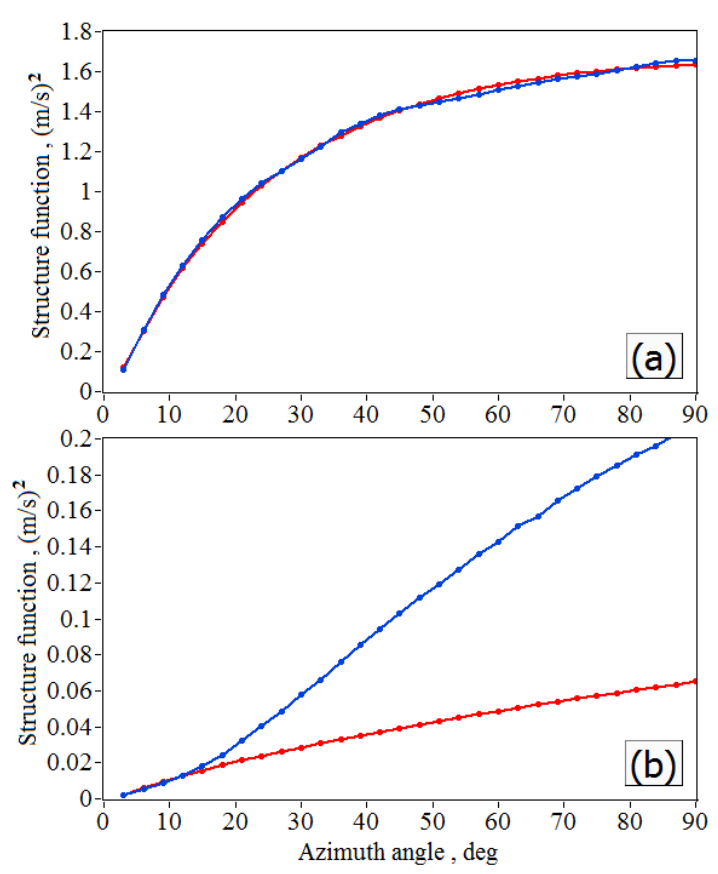

Figure 2 Structure functions $\bar{D}_{L}\left(\psi_{l}\right)$ (blue curves) and $D_{\perp}\left(l \Delta y_{k}\right) \quad$ (red curves) obtained from lidar measurements at a height of $150 \mathrm{~m}$ on 22 July 2016 at 19:00 (a) and 03:00 (b) LT.

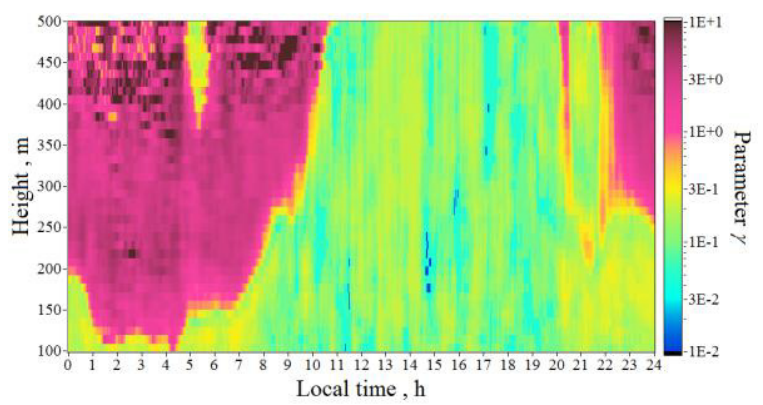

Figure 3 Spatiotemporal distribution of the parameter $\gamma$ obtained from measurements by the Stream Line lidar on 22 July 2016.

scale of turbulence, in contrast to the measurements at night, in the morning and in the evening after 20:00 LT.

\section{CONCLUSION}

Thus, in this work the feasibility of determining the wind turbulence parameters from the data measured by a conically scanning PCDL has been investigated. From the raw data of the lidar experiment carried out for three days, we obtained a spatiotemporal distribution of the turbulence parameters with a height resolution of $10 \mathrm{~m}$ (in the layer $100-500 \mathrm{~m}$ ) and a temporal resolution of $30 \mathrm{~min}$. Analysis of the experimental results has shown that the turbulence structure is well described by the von Karman model in a layer of intensive mixing. The method described in Section 2 does not work for the data measured at night (under stable temperature stratification). Possible reason is a significant effect of horizontal inhomogeneity and nonstationarity of the mean wind at a very weak turbulence.

\section{ACKNOWLEDGEMENTS}

This study was supported by the Russian Foundation for Basic Research, Project No. 1605-00668.

\section{References}

[1] Banakh, V.A., and Smalikho, I.N., 2013: Coherent Doppler Wind Lidars in a Turbulent Atmosphere, Artech House, Boston and London, 248 pp.

[2] Frehlich, R.G., Meillier, Y., Jensen, M.L., Balsley, B., and Sharman, R., 2006: Measurements of boundary layer profiles in urban environment, J. Appl. Meteorol. Clim. 45(6), 821-837.

[3] Smalikho, I.N., Banakh, V.A., 2013: Accuracy of estimation of the turbulent energy dissipation rate from wind measurements with a conically scanning pulsed coherent Doppler lidar. Part I. Algorithm of data processing, Atmos. Ocean. Opt. 26 (5), 404-410.

[4] Eberhard, W. L., Cupp, R. E., and Healy, K. R., 1989: Doppler lidar measurement of profiles of turbulence and momentum flux, J. Atmos. Ocean. Tech., 6(10), 809-819.

[5] Smalikho, I.N., Banakh, V.A., Falits, A.V., Rudi, Yu.A., 2015: Estimation of the turbulent energy dissipation rate from the data measured by the Stream Line lidar in the surface layer of atmosphere, Atmos. Ocean. Opt. 28 (10), 901905 [in Russian].

[6] Byzova, N.L., Ivanov, V.N., and Garger, E.K., 1989: Turbulence in Atmospheric Boundary Layer, Gidrometeoizdat, Leningrad, 265 pp. [in Russian]. 